\title{
STATISTICAL AND FRACTAL ANALISIS OF RANDOM HEIGHT FUNCTION
}

\begin{abstract}
Nanostructured semiconductor surfaces are commonly used for suppression of the light reflection. We prepared several kinds of surface structures on silicon substrate and analyzed the properties of the random height function used for the description of observed surface morphology. Statistical and fractal methods used in this analysis provide useful information for the optimization of the surface forming procedure. Multifractal analysis provides additional information about the surface morphology, not contained in the results of standard statistical methods. Numerical procedures used in the multifractal analysis were tested by using theoretical random height function created from large sets of Cantor numbers.
\end{abstract}

Keywords: Multifractal analysis, statistical methods, semiconductor structures.

\section{Introduction}

The properties of microstructure play an important role in fabrication of electronic and photonic devices, especially in solar cells [1]. In order to decrease the reflection losses and increase the absorption probability by light trapping in the solar cell structure random pyramidal textures are usually prepared at the semiconductor surface [2 - 8]. Pyramidal texture is formed by etching of the silicon surface and contains a large variety of surface shapes depending on the technological treatment. For characterization of the pyramidal surface morphology, the random height function $h(x, y)$ describing the height of atom above the reference plane at position $(x, y)$ can be used. The characteristics of the random height function are visually apparent but not sufficiently described by conventional measures like mean value or standard deviation. The values of the random height function $h(x, y)$ can be experimentally obtained by the electron microscopy or by the scanning probe methods with atomic resolution $[9,10]$. Experimental values of $h(x, y)$ posses a scale invariant structure. The particular kind of scale invariant structure in the experimental data $s(x)$ is defined by the power exponent $H$ in an equation for scaling of observed data $s(c x)=c^{H} S(x)$. The complex shape of the random height function can be described by the fractal methods in this case [11 - 14]. The variations in scale invariant structure can be often observed in the experimental surface images. This indicates a multifractal structure of scanned surface image that is defined by a multifractal spectrum of power law exponents rather than a single power law exponent for monofractal.

In this paper we describe results of statistical and multifractal analysis of theoretical surface generated by using Cantor numbers [15] as well as the random height function of real semiconductor structures, observed with atomic resolution.

\section{Multifractal analysis}

For the description of the random height function properties the multifractal singularity spectrum $f(\alpha)$ and generalized fractal dimension $D_{q}$ can be used. The box-counting method is often used for the multifractal analysis studied structure. The observed surface is divided into square areas with the size of side $\varepsilon$. In a selected area the probability measure is defined by

$P_{i}(\varepsilon) \sim \varepsilon^{\alpha_{i}}$

where $\alpha_{i}$ is singularity exponent. If $N(\alpha)$ is the number of surface areas in which $P_{i}$ has singularity $\alpha_{i}$ in an interval $\alpha_{i} \in(\alpha, \alpha+d \alpha)$, then multifractal singularity spectrum $f(\alpha)$ is defined by equation

$N(\alpha) \sim \varepsilon^{-f(\alpha)}$

\footnotetext{
* ${ }^{1}$ Stanislav Jurecka, ${ }^{2,3}$ Maria Jureckova

${ }^{1}$ Institute of Aurel Stodola, University of Zilina, Liptovsky Mikulas, Slovakia

${ }^{2}$ Mathematical Institute, Slovak Academy of Sciences, Bratislava Slovakia

${ }^{3}$ Catholic University in Ruzomberok, Slovak Republic

E-mail: jurecka@lm.uniza.sk
} 
Function $f(\alpha)$ can be interpreted as the fractal dimension of surface areas with singularity $\alpha$. This definition is connected with the multifractal measure described by the multifractal singularity spectrum.

For the characterization of multifractal properties of the studied surface generalized fractal dimension $D_{q}$ can be alternatively used. The $q$-th moment of the $P_{i}$ measure is defined by the equation

$$
D_{q}=\frac{1}{q-1} \lim _{q \rightarrow 0} \frac{\log \sum_{i} P_{i}(\varepsilon)^{q}}{\log \varepsilon}
$$

In case when $D_{q}$ does not depend on $q$ then the studied surface $h(x, y)$ is monofractal. By using the Legendre transformation we obtain relation of $f(\alpha)$ and $D_{q}$ in form

$$
f(\alpha)=\alpha q-(q-1) D_{q}
$$

For $q=0$ we obtain Hausdorff fractal dimension

$$
D_{0}=-\lim _{q \rightarrow 0} \frac{\log N}{\log \varepsilon}=\lim _{q \rightarrow 0} \frac{\log N}{\log 1 / \varepsilon}
$$

By using L'Hospital's rule we obtain for $q=1$

$$
\begin{aligned}
D_{1} & =\lim _{q \rightarrow 1} \frac{1}{q-1} \lim _{\varepsilon \rightarrow 0} \frac{\log \sum_{i} P_{i}^{q}}{\log \varepsilon}= \\
& =\lim _{\varepsilon \rightarrow 0} \lim _{q \rightarrow 1} \frac{\sum_{i} P_{i}^{q} \log P_{i}}{\log \varepsilon \sum_{i} P_{i}^{q}}=\lim _{\varepsilon \rightarrow 0} \frac{\sum_{i} P_{i} \log P_{i}}{\log \varepsilon}
\end{aligned}
$$

Dimension $D_{1}$ corresponds to the entropy or information dimension. Lower value of $D_{1}$ indicate higher disorder of observed random height function $h(x, y)$.

For $q=2$ we obtain correlation dimension

$D_{2}=\lim _{\varepsilon \rightarrow 0} \frac{\log P_{i}(\varepsilon)^{2}}{\log \varepsilon}$

suitable for an identification of the surface homogeneity. The higher degree of the surface homogeneity, the higher value of $D_{2}$ is observed [12, 13, 16, 17].

\section{Results and discussion}

For analysis of multifractal properties of the $h(x, y)$ function, we generated theoretical surface by using Cantor numbers. We determined values of the $h(x, y)$ function by random selections from large Cantor sets $C(n)$. Cantor sets were constructed by dividing of unit interval into three subintervals $(C(3))$, five $(C(5))$, seven $(C(7))$ and nine subintervals $(C(9))$. By the random selection (with uniform distribution) from these Cantor sets the different $h(x, y)$ functions representing surface areas of various size were constructed. Random surface generated by this way is shown in Fig. 1.

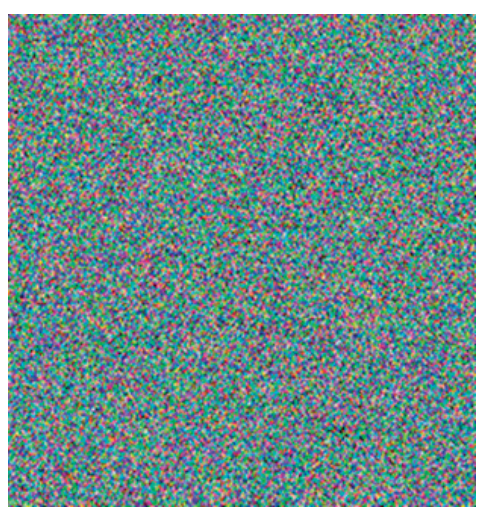

a)

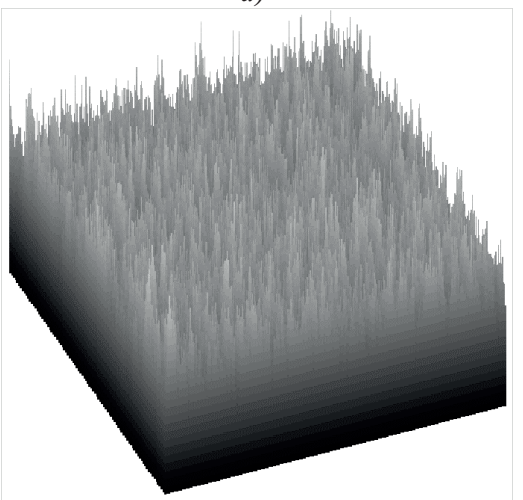

b)

Fig. 1 Random height function generated from Cantor set numbers $C(3)$; a) $2 \mathrm{D}$ plot, b) $3 \mathrm{D}$ plot

For computation of the $P_{i}$ in (Equation 1) we used the boxcounting method

$P_{i j}=\frac{h_{i j}}{\sum h_{i j}}$

where $h_{i j}$ is the mean value of the $h(i, j)$ function in the box box $(i, j)$. By using this probability we computed generalized fractal dimension $D_{q}$ (Equation 3) and multifractal spectrum $f(\alpha)$ (Equation 4). The results of developed numerical procedures show expected behaviour for multifractal structures of theoretical Cantor test surfaces and were used for analysis of real semiconductor surfaces. In Fig. 2 two distributions of pyramidal textures formed on flat silicon surface are shown. In pyramidal structure $\mathrm{d} 1$ a quasi homogeneous distribution of pyramidal shapes was formed whereas in structure $\mathrm{d} 2$ dominant fraction of small pyramids with random occurrence of very high pyramids was created. 


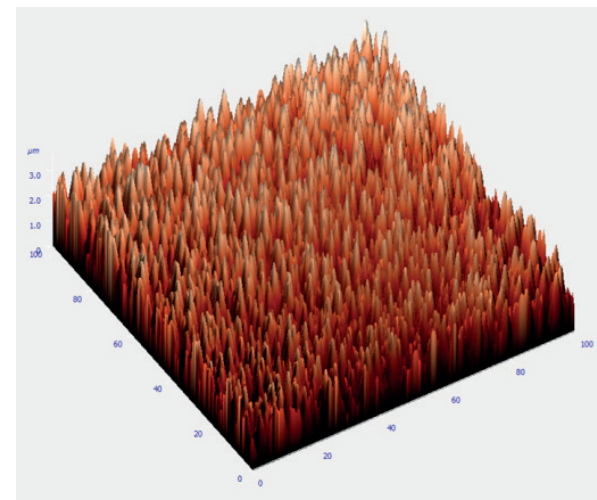

a)

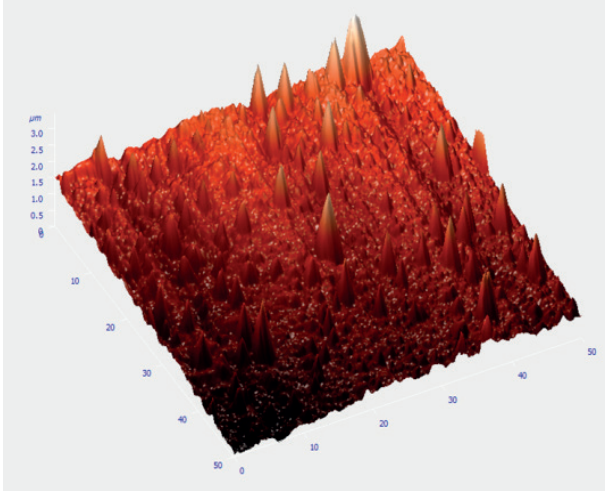

b)

Fig. 2 Pyramidal structures prepared on Si surface: a) distribution $d 1$, b) distribution $d 2$

Statistical characteristics of the random height function $h(i, j)$ values obtained by the atomic force microscopy experiment for these pyramidal structures are shown in Table 1.

Statistical characteristics of the experimental random height function

Table 1

\begin{tabular}{lccc}
\hline Distribution & & d1 & d2 \\
\hline Max & $\mathrm{nm}$ & 2946.51 & 2206.76 \\
Peek-to-peek $S_{y}$ & $\mathrm{~nm}$ & 2946.51 & 2206.76 \\
10-point height $S_{z}$ & $\mathrm{~nm}$ & 1483.12 & 1069.98 \\
Average roughness $S_{\alpha}$ & $\mathrm{nm}$ & 1417.75 & 664.146 \\
Mean square roughness $S_{q}$ & $\mathrm{~nm}$ & 461.827 & 146.431 \\
Coefficient of variation $S_{q} / S_{\alpha}$ & & 0.326 & 0.22 \\
Skewness $S_{s k}$ & & 0.154 & 2.467 \\
Kurtosis $S_{k u}$ & & -0.389 & 11.761 \\
\hline
\end{tabular}

The values of these statistical characteristics can be used in study of the surface roughness properties. Coefficient of variation is a relative measure of variability between structures with different averages. For distribution $\mathrm{d} 1$ this indicates higher surface roughness. Higher value of the skewness for distribution d2 is influenced by higher inhomogeneity of the pyramidal shapes in this distribution (dominant fraction of small pyramids). Negative value of kurtosis for distribution d1 shows low influence of different pyramidal shapes onto the statistical properties of d1 distribution and high value of kurtosis for distribution $\mathrm{d} 2$ indicates the presence of abnormally high pyramidal shapes at observed surface.

Statistical analysis provides useful information about the random height function properties. Supplementary information can be obtained by using multifractal methods. In Fig. 3 results of multifractal analysis of four pyramidally textured surfaces are shown.

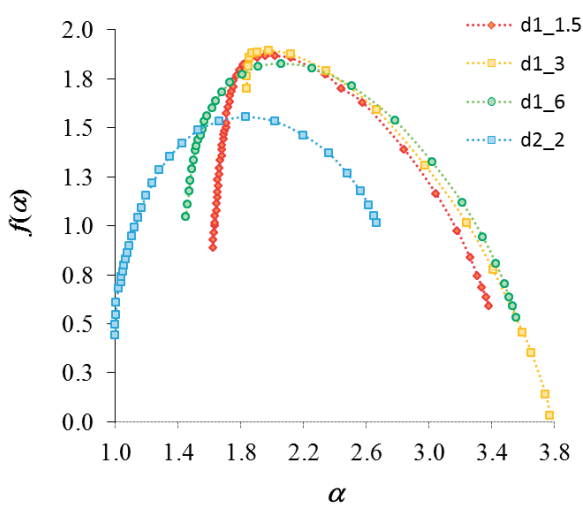

a)

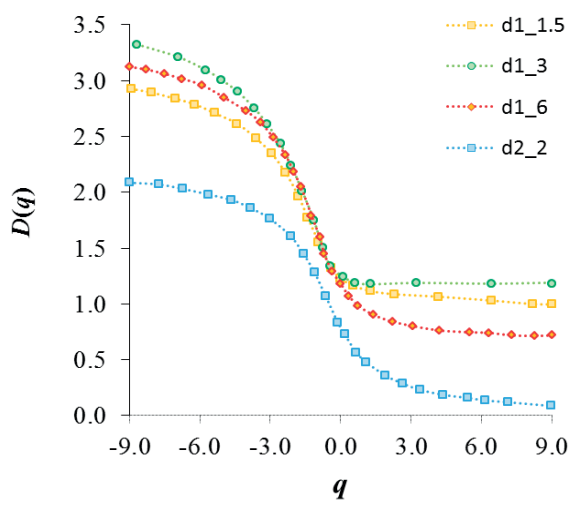

b)

Fig. 3 Results of multifractal analysis of pyramidal texture with maximal heights of pyramids $1.5,1.3,1.6$, and $2.2 \mu \mathrm{m}$ :

a) multifractal singularity spectrum $f(\alpha)$,

b) generalized fractal dimension $D(q)$

We analyzed distributions $\mathrm{d} 1$ and $\mathrm{d} 2$ where maximal height of the pyramid equals $3 \mu \mathrm{m}$ and we studied also distribution d1 with maximal height of pyramidal shapes $6 \mu \mathrm{m}$ and $1.5 \mu \mathrm{m}$. Generalized fractal dimension $D_{q}$ curves show decreasing trend significant for multifractal surface morphology. In plot of the $D_{q}$ curves we observe important difference between characteristics for $\mathrm{d} 1$ and $\mathrm{d} 2$ distribution. Surface structure $\mathrm{d} 2$ has significantly 
lower $D_{q}$ values in comparison to homogeneous distribution d1 for all $q$ values. Value of dimension $D_{0}$ connected with traditional fractal dimension is very similar for all pyramidal distributions (with different heights of pyramids). On the other side significant differences can be seen in comparison of distributions $\mathrm{d} 1$ and $\mathrm{d} 2$. Lower value of dimension $D_{1}$ for distribution d2 indicates higher disorder in this pyramidal distribution (higher randomness) in comparison to d1 distribution. Maximal value of the correlation dimension $D_{2}$ indicates the highest homogeneity of the observed surface.

Multifractal characteristics are therefore very sensitive to small changes in the surface height function and in the distribution of pyramidal shapes. Different shapes of the $D_{q}$ curves enable us to distinguish reliably between the properties of different distributions $\mathrm{d} 1$ and $\mathrm{d} 2$ as well as between small changes in the same distribution of pyramidal shapes (for example, for distributions type $\mathrm{d} 1$ with different heights of pyramids). The obtained results can be therefore used for the optimization of technological steps of surface texture forming procedure.

Multifractal singularity spectrum $f(\alpha)$ has concave shape typical for multifractal morphology. The maximal height of the $f(\alpha)$ spectrum is given by the $D_{0}$ value and the width of $f(\alpha)$ spectrum corresponds to the variability of shapes of the morphological objects. Symmetry of the $f(\alpha)$ curve is different for distribution $\mathrm{d} 2$, which indicates higher non-uniformity of distribution $\mathrm{d} 2$ in comparison to distribution $\mathrm{d} 1$.

In Fig. 4 multifractal spectrum $f(\alpha)$ curves for thin nanocrystalline layers formed by etching of silicon surface in the HF acid in contact with the Pt electrode are shown.

The analyzed structures were etched for 10, 20, and 30 seconds and values of the random height function were obtained by the scanning electron microscope with magnification 2000 . From the shape of $f(\alpha)$ curves we can see, that the surface morphology develops primarily in the first stages of etching procedure (during the first 20 seconds). With the prolongation of etching time the development of surface morphology stabilizes.

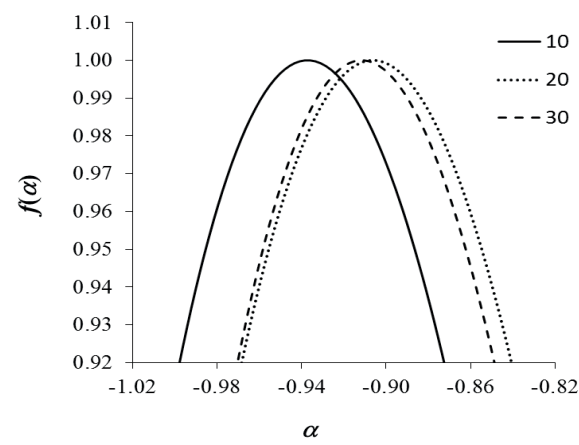

Fig. 4 Multifractal spectrum $f(\alpha)$ curves of the nanocrystalline Si layers

\section{Conclusions}

Statistical and multifractal analysis provides information about the shape of morphological objects development as well as about the intensity of modification of treated surface morphology. Multifractal analysis is a suitable tool for study of the random height function properties, providing additional information about the surface morphology, not contained in the results of standard statistical methods.

\section{Acknowledgements}

This work was supported by grant of Science and Technology Assistance Agency APVV-15-0152, Scientific Grant Agency VEGA 2/0031/15 and VEGA 1/0076/15, Centre of Excellence of Power Electronics Systems and Materials II - ITMS 26220120046 , project ITMS 26210120021, and Japan Society for the Promotion of Science.

\section{References}

[1] MUlleroVA, J., SUTTA, P., JURECKA, S.: Thin Film Silicon in Photovoltaics: The Role of Structure and Microstructure. Communications - Scientific Letters of the University of Zilina, 8(1), 5-9, 2006.

[2] ANGermanN, H., RAPPICH, J., KORTE, L., SIEBER, I., CONRAD, E., SCHMIDT, M., HUbENER, K., POLTE, J., HAUSCHILD, J.: Wet-Chemical Passivation of Atomically Flat and Structured Silicon Substrates for Solar Cell Application. Applied Surface Science, 254, 3615-3625,2008.

[3] ANGERMAnN, H., CONRAD, E., KORTE, L., RAPPICH, J., SCHUlZE, T. F., SCHMIDT, M.: Passivation of Textured Substrates for a-Si:H/c-Si Hetero-junction Solar Cells: Effect of Wet-Cchemical Smoothing and Intrinsic a-Si:H Interlayer. Materials Science and Engineering: B, 159-160, 219-223, 2009. 
[4] STEGEMANN, B., KEGEL, J., MEWS, M., CONRAD, E., KORTE, L., STURZE-BECHER, U., ANGERMANN, H.: Passivation of Textured Silicon Wafers: Influence of Pyramid Size Distribution, a-Si:H Deposition Temperature, and Post-treatment. Energy Procedia, 38, 881-889, 2013.

[5] TAKAHASHI, M., FUKUSHIMA, T., SEINO, Y., KIM, W.-B., IMAMURA, K., KOBAYASHI, H.: Surface Structure Chemical Transfer Method for Formation of Ultralow Reflectivity Si Surfaces. Journal of Electrochemical Society, 160, H443-H445, 2013.

[6] IMAMURA, K., FRANCO JR., F. C., MATSUMOTO, T., KOBAYASHI, H.: Ultra-Low Reflectivity Polycrystalline Silicon Surfaces Formed by Surface Structure Chemical Transfer Method. Applied Physics Letters, 103, 3110-1-3110-4, 2013.

[7] FUKUSHIMA, T., OHNAKA, A., TAKAHASHI, M., KOBAYASHI, H.: Fabrication of Low Reflectivity Poly-Crystalline Si Surfaces by Structure Transfer Method, Electrochem. Solid-State Letters, 14, B13-B15, 2011.

[8] JURECKA, S., ANGERMANN, H., KOBAYASHI, H., TAKAHASHI, M., PINCIK, E.: Multifractal Analysis of Textured Silicon Surfaces. Applied Surface Science, 301, 46-50, 2014.

[9] JURECKA, S., JURECKOVA, M., KOBAYASHI, H., TAKAHASHI, M., MADANI, M., PINCIK, E.: Statistical and Fractal Properties of Semiconductor Surface Roughness. Advances in Electrical and Electronic Engineering, 7(1-2), 377-381, 2008.

[10] JURECKA, S., KOBAYASHI, H., TAKAHASHI, M., MATSUMOTO, T., JURECKOVA, M., CHOVANEC, F., PINCIK, E.: On the Influence of the Surface Roughness onto the Ultrathin $\mathrm{SiO}_{2} /$ Si Structure Properties. Applied Surface Science, 256, 5623-5628, 2010.

[11] GOLLION, P., GREENE, G.: Determination of Fractured Steel Surface Roughness by Atomic Force Microscopy Using Fractalbased Approaches, Surface and Interface Analysis, 24, 282, 1996.

[12] HOSSEINABADI, S., MORTEZAALI, A., MASOUDI, A. A.: Investigating Aluminum Thin Films Properties by Stochastic Analysis. Surface and Interface Analysis, 40, 71, 2008.

[13] TENG, H. T., EWE, H. T., TAN, S. L.: Multifractal Dimension and Its Geometrical Terrain Properties for Classification of MultiBand Multi-Polarized Sar Image. Progress in Electromagnetics Research, 104, 221-237, 2010.

[14] ANNADHASON, A.: Methods of Fractal Dimension. International Journal of Computer Science and Information Technology \& Security, 2(1), 166-169, 2012.

[15] CHOVANEC, F.: Cantor Sets. Science \& Military, 5(1), 5-10, 2010.

[16] HARTE, D.: Multifractals: Theory and Applications. Chapman and Hall/CRC, Boca Raton, 2001.

[17] ChHABRA, A. B., MENEVEAU, C., JenSEN R. V., SREENIVASAN K. R.: Direct Determination of the $f(\alpha)$ Singularity Spectrum and its Application to Fully Developed Turbulence. Physical Review, 40(9), 5284-5294, 1989. 